\title{
Nonlinear distortion in a microwave high power amplifier
}

\author{
Bezoušek Pavel \\ Department of Electrical Engineering \\ Faculty of Electrical Engineering and \\ Informatics, University of Pardubice \\ Pardubice, Czech Republic \\ Pavel.Bezousek@upce.cz
}

\author{
David Matoušek \\ Department of Electrical Engineering \\ Faculty of Electrical Engineering and \\ Informatics, University of Pardubice \\ Pardubice, Czech Republic \\ David.Matousek@upce.cz
}

\author{
Luboš Rejfek \\ Department of Electrical Engineering \\ Faculty of Electrical Engineering and \\ Informatics, University of Pardubice \\ Pardubice, Czech Republic \\ Lubos.Rejfek@upce.cz
}

\begin{abstract}
In this paper the high-power amplifier CGHV31500F designed for the radar S-Band application is studied. The amplifier module is based on GaN HEMT encapsulated internally matched transistor, delivering more than $500 \mathrm{~W}$ in a pulse regime. Nonlinear model of this amplifier was developed and nonlinear distortion of a standard radar signal and of possible future QAM signals are predicted.
\end{abstract}

Keywords-Nonlinear Amplifier, Characteristics of the HighPower Amplifier (HPA), Nonlinear model HPA

\section{INTRODUCTION}

Ground based medium and long-range radars transmit usually $10-30 \mathrm{~kW}$ of pulse power in pulses of 10 to $100 \mu \mathrm{s}$. The solid state pulsed high power RF amplifiers in the radar transmitter consist of multiple parallel modules producing 0.1 $-1 \mathrm{~kW}$ each. The parameters of the whole transmitter are then determined basically by those high-power amplifier (HPA) modules. Using mostly frequency or phase modulated signals i.e. constant envelope signals radars usually do not suffer from HPA nonlinearity effects. Nevertheless, in extremely broadband systems or in multi radar or multi transmitter systems like MIMO radars also amplitude modulation could be incorporated. Then also nonlinear characteristics of the HPA modules become important. To preserve a good quality of the radar signal, necessary for target detection, some linearization procedure in the transmitter is needed and hence a mathematical model of the particular HPA for system simulation is plausible.

A great number of various nonlinear models of RF HPA were developed and described in publications ([1] - [5], [9], [11], [13] - [16]), together with various model identification procedures. The best insight to the nonlinearity mechanisms offer physical based models like [1] or [2], requiring detailed knowledge on the particular device construction, which is rarely available. The most accurate results provide models based on Volterra series but with demand of complicated identification, usually not possible during system operation. Also, linearization means are frequently described in the literature. For HPA mostly predistortion linearizers are proposed (e.g. [7] - [10]) with only amplitude or phase/amplitude feedback adaptivity. It is caused mainly by communication systems needs where instantaneous correction of nonlinear effects is appropriate. In the case of radars transmitters, the linearization was not of great concern up to now due to the mentioned use of constant envelope modulations. Furthermore, the GaN HEMT based amplifiers operating in $\mathrm{AB}$ regime provide only a weak nonlinearity ([1], [2]). But having in mind the emerging MIMO and other multi transmitter radar systems certain adaptive linearization for

The described research was supported by the projects of the Czech Ministry of Industry and Trade No. FV10484 and FV 20701. radar HPA would be desirable with a simple and sufficiently accurate HPA nonlinear model. In this paper the parametrical model of the CGHV31500F HPA, based on nonlinear amplifier characteristics measurement is introduced and the nonlinearity impact on various signals is presented.

\section{NONLINEAR AMPLIFIER CHARACTERISTICS}

The amplifier output power on input power dependence measured at frequencies in the $2.7-3.1 \mathrm{GHz}$ band is shown in the Fig. 1. The measurements were implemented in the pulse mode with the pulse length of $1 \mu$ s and the pulse repetition frequency of $1 \mathrm{kHz}$. The input signal amplitude was stabilized during the pulse in the range of $\pm 0.2 \mathrm{~dB}$, the amplifier supply regime was $\mathrm{CW}$. According to the producer's Application Notes [12] the amplifier operates in the $\mathrm{AB}$ mode what corresponds to the measured power transfer characteristics. Regarding the AM/PM characteristics we will refer to previous works of other authors ([1], [2],[3]).

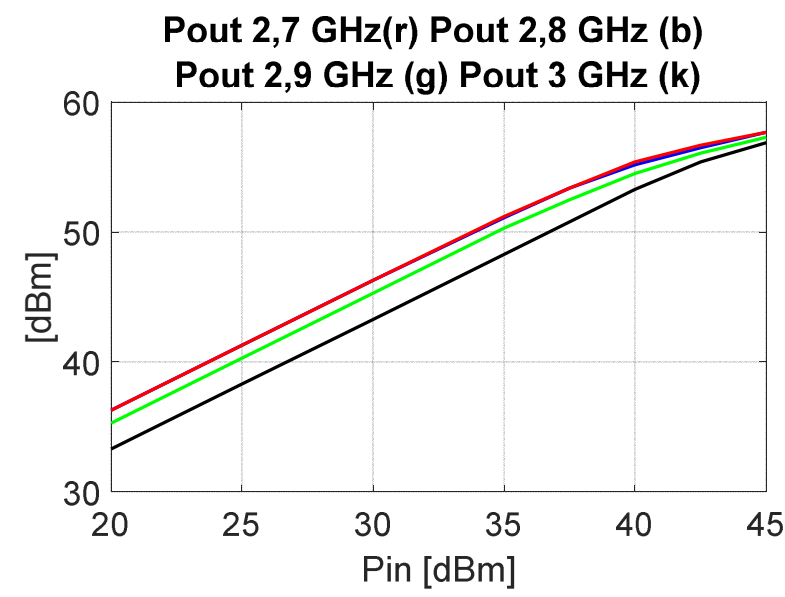

Fig. 1. The measured power transfer characteristics of the amplifier.

Weak power transfer nonlinearity together with its small frequency dependence allows us to represent the power amplifier using the Wiener model of a dynamic nonlinear system ([13],[14],[15]) consisting of a linear time invariant (LTI) circuit with the pulse response $h(t)$ and a memoryless nonlinearity with $f(u)$ response (see Figure 2 ).

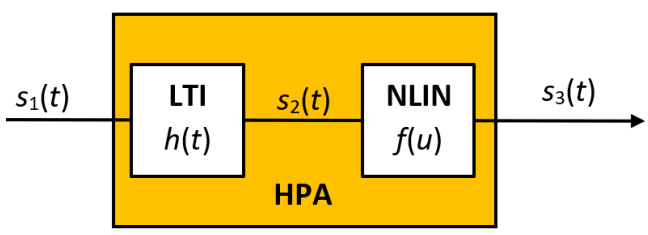

Fig. 2. Nonlinear model of high power amplifier HPA. 
Here:

$$
\begin{aligned}
& s_{2}(t)=h(t)^{*} s_{1}(t) \\
& s_{3}(t)=f\left(s_{2}(t)\right) .
\end{aligned}
$$

The LTI was modeled by a simple resonant circuit with a transfer function $H(\omega)$ and the nonlinearity function $f(u)$ by a 7 th order polynomial with only odd powers of $u$ :

$$
\begin{gathered}
H(\omega)=\frac{1}{1+2 \mathrm{j} \frac{\omega-\omega_{0}}{2 \pi B}} \\
f(u)=\sum_{k=1}^{4} a_{2 k-1} u^{2 k-1} .
\end{gathered}
$$

Using the described model, the measured output power on input power and frequency dependence was approximated with a reasonable accuracy (see figure 3 ).

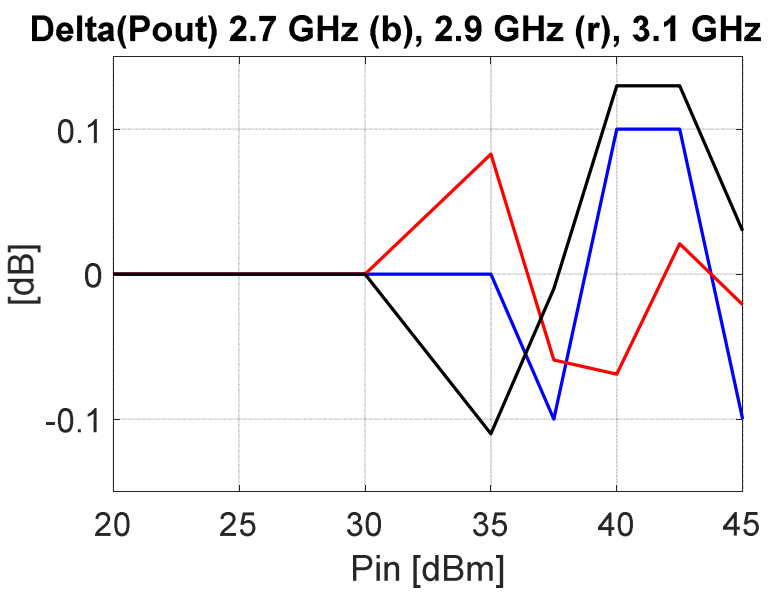

Fig. 3. Differences between measured and modeled PA output power.

We had not realized our own AM/PM distortion measurement on the power amplifier in question. But other authors in their works ([1], [2], [5], [7]) showed, that the amount of the phase transfer error due to the nonlinearity at microwave $\mathrm{GaN}$ based power amplifier is of only $10^{\circ}$ to $20^{\circ}$ for the power range till the hard limitation occurs. Generally, the phase distortion in field effect transistors is due to nonlinearities of the gate-source capacitance, the drain-source and the drain to gate feedback capacitance. The feedback capacitance reduces the effect of the other ones. As the drain capacitance of the high voltage HEMT is quite low, only gate to source and drain to gate capacitances operating in opposite are effective. That is why the phase distortion in $\mathrm{GaN}$ based power amplifiers are quite small ([1]). On the other hand, the HPA based on GaN transistors are subjects to memory effects due to electron traps, thermal effects or a bias circuit. These effects are rather slow and usually do not interfere with the signal modulation, but they can seriously affect results of static measurements of the amplifier nonlinearity. Nevertheless, in order to explore also a possible interference of the AM/PM distortion on the amplified signals we simulated it as an amplifier group delay $\tau_{\mathrm{g}}$ dependence on the signal amplitude. Based on the measurement of [1], [2] and [16] we roughly estimated the power induced group delay change as a piecewise linear-quadratic function shown at the figure 4.

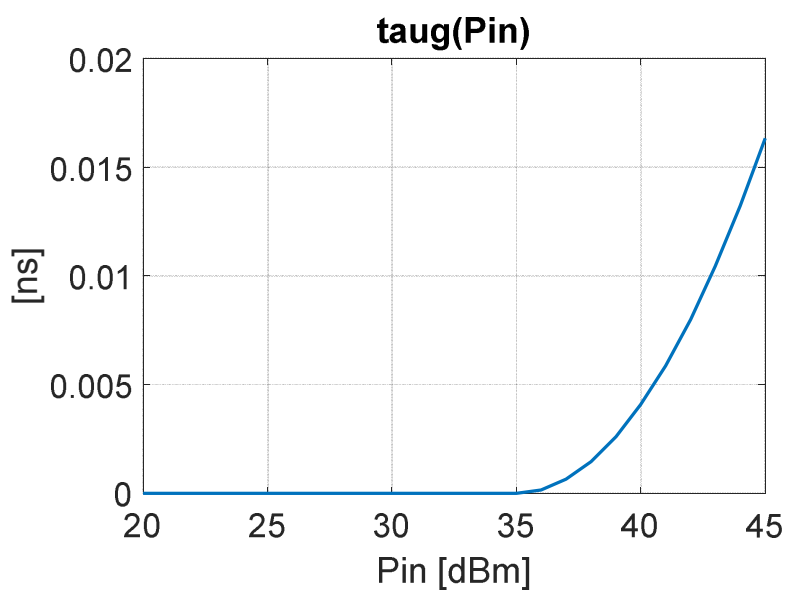

Fig. 4. Estimated amplifier group delay on input power dependence.

\section{THE AMPLIFIER NONLINEARITY EFFECTS ON PULSED FREQUENCY MODULATED SIGNALS}

Using the described model, we have tested the effect of the nonlinearity on pulsed frequency modulated radar signals (LFM) and on 16 QAM and 64 QAM signals with the parameters, shown in the TABLE I. The maximum input power was $25 \mathrm{~W}$ and the center frequency was $2.9 \mathrm{GHz}$ in all cases.

TABLE I. PARAMETERS OF THE TESTING SIGNALS

\begin{tabular}{|c|c|c|c|c|}
\hline \multirow{2}{*}{ Parameter } & \multicolumn{4}{|c|}{ Modulation } \\
\cline { 2 - 5 } & LFM1 & LFM2 & 16-QAM & 64-QAM \\
\hline Pulse length & $80 \mu \mathrm{s}$ & $0.4 \mu \mathrm{s}$ & $80 \mu \mathrm{s}$ & $80 \mu \mathrm{s}$ \\
\hline Bandwidth & $1.25 \mathrm{MHz}$ & $250 \mathrm{MHz}$ & $1.25 \mathrm{MHz}$ & $1.25 \mathrm{MHz}$ \\
\hline $\begin{array}{c}\text { Number } \\
\text { of samples }\end{array}$ & 150 & 150 & 150 & 250 \\
\hline
\end{tabular}

The effect of distortion on the pulsed frequency modulated radar signals were checked using the matched compression filter and no effects on the signal at the filter output were detected in this case. The only effects of the nonlinear distortion in the high-power amplifier was generation of higher harmonics. The described model prediction of the harmonic generation is shown at the Fig. 5. Thought they do not hinder the signal processing in the radar, they are generally unacceptable due to their interference of other electromagnetic spectrum users and therefore they should be removed using a special harmonic suppression filter at the HPA output.

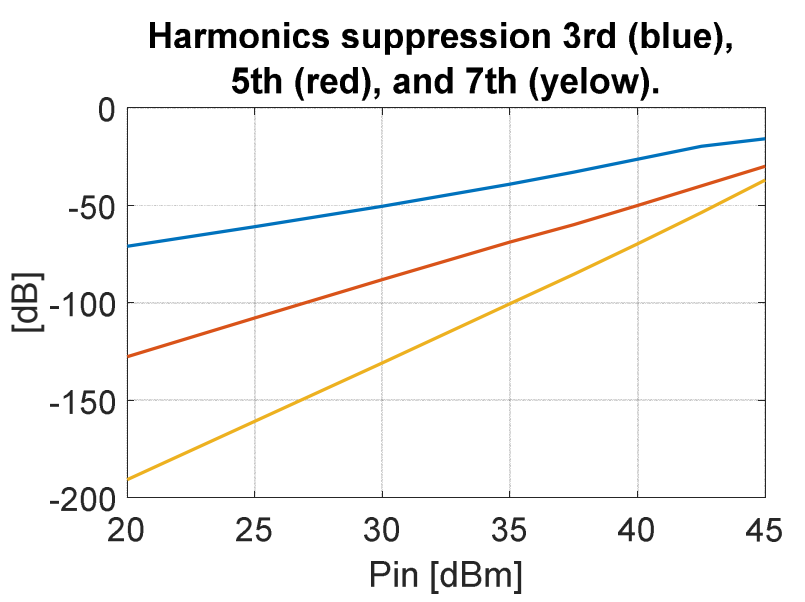

Fig. 5. Harmonics generated in the studied power amplifier - model prediction. 
As was mentioned above certain modern applications, like MIMO radar systems, need not only a suppression of the signal (time) sidelobes after pulse compression in the receiver but also cross-correlation minimization between signals of individual transmitters in the MIMO system. This new requirement on signal orthogonality simultaneously with the common demand on low sidelobes is difficult to achieve using only PSK or other constant-envelope modulations or it leads to serious deterioration of the both qualities ([17], [18]). Hence addition of amplitude modulations like in the QAM or OFDM modulations could bring a further degree of freedom for this application. But the incorporation of the amplitude modulations leads to great distortion of the transmitted signal as can be seen from the figure 6, where the pulsed 16 QAM signal (table 1) was subjected to the distortion, described by our model i.e. by relations (1), (2), (3) and (4). This result calls for the transmitter linearization.

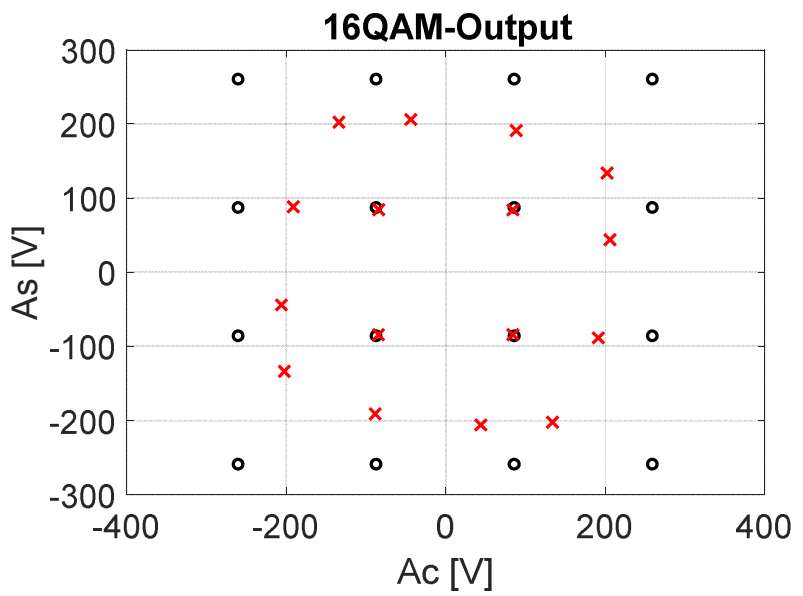

Fig. 6. Comparison of the modeled distorted 16QAM output signal (red crosses) with the undistorted one (black circles).

\section{LINEARIZATION}

In order to suppress the nonlinear effects of high-power amplifiers on amplitude modulated communication signals in changing environment, various types of adaptive linearizers were developed. Recently adaptive digital pre-distortion (DPD) linearizers are used frequently in this function (e.g. ([3], [5], [7], [10]). In these linearizers the corrections are not applied to the amplifier input signal but directly to the symbols at the complex envelope (IQ) level. To check the application of this technology to the radar pulsed QAM signal we verified its operation using a model of the DPD linearizer according to the diagram in the figure 7. Discrete time series of data symbols $\{m(k), n(k)\}_{k}$ enter the pre-processor, where the actual complex amplitude components $I(k)$ and $Q(k)$ are computed in real time and delivered to the modulator. The pulsed QAM signal is amplified in the HPA and the amplified and the distorted signal is compared with the delayed input signal. Actual $P_{\text {out }}\left(P_{\text {in }}\right)$ and $\Delta \Phi_{\text {out }}\left(P_{\text {in }}\right)$ dependences are then evaluated. Here Pout is an instantaneous power and $\Delta \Phi_{\text {out }}$ is a phase error of the output signal. By the phase error we mean the difference between the output and input signal phase. Then the new required powers $P_{i n}(m, n)$ and the needed phase corrections $\Delta \Phi(m, n)$ of the individual QAM symbols, related to the data symbols $(m, n)$ and the corresponding corrected complex amplitude components $I(m, n)$ and $Q(m, n)$ are computed and supplied to the IQ modulator.

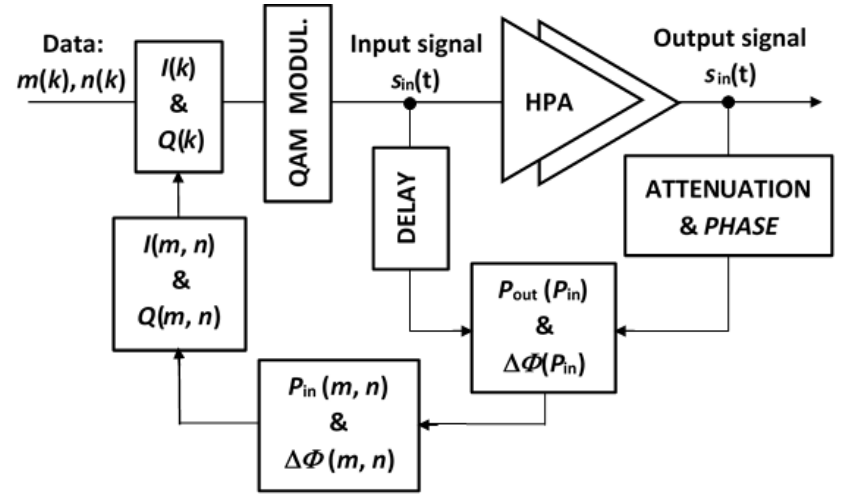

Fig. 7. Diagram of the HPA with the proposed QAM linearizer.

The results of this linearization operation, with HPA nonlinearity, based on the described model are shown in the constellation diagram at the figure 8 . The circles represent the ideal positions of the 16QAM symbols at the HPA output and the crosses the symbol positions when the nonlinear HPA is used with the linearizer. A considerable improvement in positions agreement is apparent comparing it with the fig. 6 , where the signal without linearization is displayed.

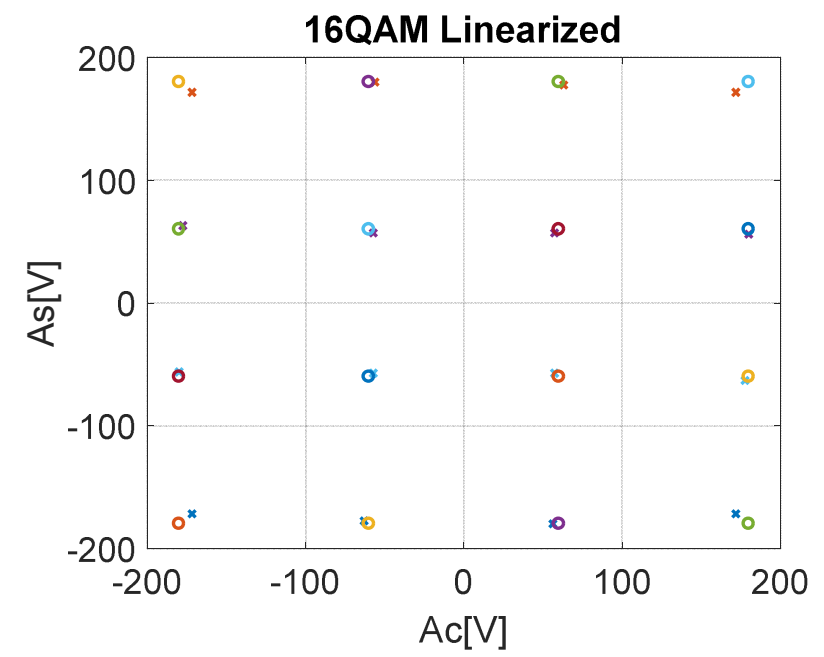

Fig. 8. Comparison of the ideal (circles) and modeled distorted but linearized (crosses) output 16 QAM signal.

\section{CONCLUSION}

In the paper a simple model of RF high power amplifier nonlinearity was designed based on measurement of the $P_{\text {out }}\left(P_{\text {in }}\right)$ dependence. The group delay on the output power dependence as a source of the AM/PM distortion was estimated from measurements and modeling of other authors. It was shown, that these mechanisms have only small effects on standard radar frequency modulated pulsed signals. That is why no linearization measures are needed in the usual situation. The most noticeable effect of the amplifier nonlinearity in the standard radar is harmonic generation, which could be suppressed using a harmonic rejection filter.

In the case the modulations will include the amplitude modulation (possibly in the future MIMO systems) the linearization of $\mathrm{AM} / \mathrm{AM}$ and $\mathrm{AM} / \mathrm{PM}$ will be necessary and then the existence of a simple and accurate parametric model of HPA will be plausible. The effect of a digital pre-distortion linearizer to the HPA with the described nonlinearity dealing with a 16QAM modulation was checked using the described 
model and a considerable improvement over the nonlinearized case was observed.

\section{ACKNOWLEDGEMENT}

The described research was supported by the projects of the Czech Ministry of Industry and Trade No. FV10484 and FV 20701

\section{REFERENCES}

[1] L.C.Nunes, P.M.Cabral, J.C.Pedro, AM/AM and AM/PM Distorsion Generation Mechanism in Si LDMOS and GaN HEMT Based RF Power Amplifiers, IEEE Transactions on MTT, Vol. 62, No 4, Apr. 2014, pp $799-809$

[2] P.M.Cabral, J.C.Pedro, N.B.Cavalho, Dynamic AM-AM and AM-PM Behavior in Microwave PA Circuits, In: Asia-Pacific Microwave Conference 2005 Proceedings, Suzhou, China, Dec. 2005

[3] M. Nakayama, K. Mori, K. Yamauchi, Y Itoh, T. Takagi, A Novel Amplitude and Phase Linearizing Technique for Microwave Power Amplifiers, In: Proc. Of the 1995 IEEE MTT-S International Microwave Symposium, Orlando, USA, May 1995, pp. 1451 - 1454

[4] A. Wentzel, F. Hühn, W. Heinrich, The Digital Power Amplifier for Wireless Infrastructure: Status and Prospects, In: Proc. of the 2017 IEEE Topical Conf. on RF/Microwave Power Amplifiers for Radio and Wireless Application, Phoenix, USA, Mar. 2017, pp. 14 - 17.

[5] A. Katz, R. Dorval, Linearizing high power amplifiers (with emphasis on predistorsion and GaN devices), In: 2012 IEEE Topical Conf. on RF/Microwave Power Amplifiers for Radio and Wireless Application, Santa Clara, USA, Jan. 2012, pp. 33 - 36

[6] A. Al Muhaisen, P. Wright, J.Lees, P.J.Tasker, S.C Cripps, J. Benedict, Novel Wide Band High-Efficiency Active Harmonic Injection Power Amplifier Concept, In: 2010 IEEE MTT-S International Microwave Symposium, Anaheim, USA, May 2010, pp. $664-667$.

[7] T. Ota, H. Ishikawa, K. Nagatani, A Novel Adaptive Digital Predistortion for Wideband Power Amplifiers With Memory Effects, In: 2015 Asia - Pacific Microwave Conference, Nanjing, China, Dec. 2015, pp.

[8] X. Lingjun, W. Xiaoguang, W. Qiang, Z. Ping, Adaptive Digital Predistorter for Radio Frequency Power Amplifier Linearizator, In IEEE 2007 Int. Symposium on Antennas and Propagation and EMC
Technologies for Wireless Communications, Aug. 2007, pp. $1390-$ 1393

[9] P.M. Asbeck, H. Kobayashi, M. Iwamoto, G.Hannington, S. Nam, L.E.Larson, Augmented Behavioral Characterisation for Modeling Nonlinear Response of Power Amplifiers, In: 2002 IEEE International MTT-S Digest, June 2002, pp. 135 - 138

[10] B. Shi, S.W.Leong, B. Luo, W. Wang, A Novel GHz-Bandwidth RF Predistortion Linearizer for Ka Band Power Amplifier, In: TNCON 2017 IEEE Conference, Penang, Malaysia, Nov. 2017, pp. 1610-1613.

[11] S. P. Yadav, S.C. Bera, Linearity improvements of microwave power amplifiers, In: 2016 IEEE Annual India Confrence INDICON, Bangalore, India, Dec. 2016, pp.

[12] CGHV31500F Data sheet, Cree Inc., available at: $\mathrm{http}: / /$ www.alldatasheet.com/datasheetpdf/pdf/799233/CREE/CGHV31500F.html

[13] Kibangou A. Y., Favier G., Wiener -Hammerstein systems modeling using diagonal Volterra kernels coefficients, IEEE Signal Processing Letters, Vol 13, Issue 6, June 2006, pp. 381 - 384

[14] Billings S., Fakhouri S., Identification of systems containing linear dynamics and static nonlinear elements, Automatica, vol. 18, 1982, pp. $15-26$.

[15] Gilabert P., Montoro G., Bertran E., On the Wiener and Hammerstein models for power amplifier predistorsion, In: 2005 Asia- Pacific Microwave Conference, Suzhou, China, March 2006

[16] Dunleavy L., Tranzistor and Amplifier Modeing Methods for Microwave Design, University of South Florida, Tampa, FL., Dec. 2006

available: https://www.ieee.li/pdf/viewgraphs/transistor_amplifier_m odeling_methods_for_microwave_design.pdf

[17] Griep K., R., Ritcey J., A., Burlingame J., J., Polyphase Codes and Optimal Filters for Multiple User Ranging, IEEE Trans. on AES-Vol. 31, No2, Apr.1995, pp. $752-767$

[18] Daum F., Huang J., MIMO Radar: Snake Oil or a Good Idea?, 2009 International Waveform Diversity and Design Conference, Orlando, Florida, Feb. 2009

[19] Zhu A., Brazil T. J., An adaptive Volterra predistorter for the linearization of RF high power amplifier, MTT-IMS 2002, Vol.1, pp. $461-464,2002$ 